EPJ Web of Conferences 79, 02007 (2014)

DOI: $10.1051 /$ epjconf/20147902007

(C) Owned by the authors, published by EDP Sciences, 2014

\title{
A Robotic arm for optical and gamma radwaste inspection
}

\author{
L. Russo ${ }^{1}$, L. Cosentino ${ }^{1}$, A. Pappalardo ${ }^{1}$, M. Piscopo ${ }^{1}$, C. Scirè ${ }^{1}$, S. Scirè ${ }^{1}$, G. Vecchio ${ }^{1}$, G. Muscato ${ }^{2}$ \\ and P. Finocchiaro ${ }^{1}$ \\ ${ }^{1}$ INFN Laboratori Nazionali del Sud, via S. Sofia 62, 95125 Catania, Italy \\ ${ }^{2}$ DIEEI, Università di Catania, via S. Sofia 64, Catania, Italy
}

\begin{abstract}
We propose Radibot, a simple and cheap robotic arm for remote inspection, which interacts with the radwaste environment by means of a scintillation gamma detector and a video camera representing its light $(<1 \mathrm{~kg})$ payload. It moves vertically thanks to a crane, while the other three degrees of freedom are obtained by means of revolute joints. A dedicated algorithm allows to automatically choose the best kinematics in order to reach a graphically selected position, while still allowing to fully drive the arm by means of a standard videogame joypad.
\end{abstract}

\section{Introduction}

The term radioactive waste (or shortly radwaste) indicates waste containing radioactive material. It is usually produced as a residue of nuclear processes, such as nuclear fission, even though industrial processes and medical applications may also produce radioactive waste. The only viable solution proposed so far to the problem posed by the radioactive waste produced all over the world, is packing it into special drums to be segregated into suitable storage sites, for short and medium term radwaste, or into so called geological burial sites for long-term radwaste.

The DMNR system (Detector Mesh for Nuclear Repositories) [1], developed at INFN-LNS within the framework of the INFN-E strategic project, aims at the remote on-line monitoring of radwaste drums in the short and medium term storage sites, in order to minimize the direct interaction of human operators with radwaste and to provide a real time overview of the activity around each single radwaste drum [2]. It is based on a low cost scintillating fiber, replicated to compose a grid around each drum. A SiPM (Silicon PhotoMultipier [3]) allows to count gamma radiation and to acquire informations in order to send them on-line for a public reference.

In this context robotics aims at creating solutions and tools that can improve the inspection in the storage sites. Several robotic systems have already been developed during recent years for this purpose, however all of them are typically rather complex and quite expensive.

\section{Manipulator design}

The reason for the development of a robotic system, as already mentioned, is the reduction of humanradwaste interaction. The possibility to use a manipulator decreases the inspection time and increases the number of inspected areas.

This is an Open Access article distributed under the terms of the Creative Commons Attribution License 4.0, which permits unrestricted use, distribution, and reproduction in any medium, provided the original work is properly cited. 


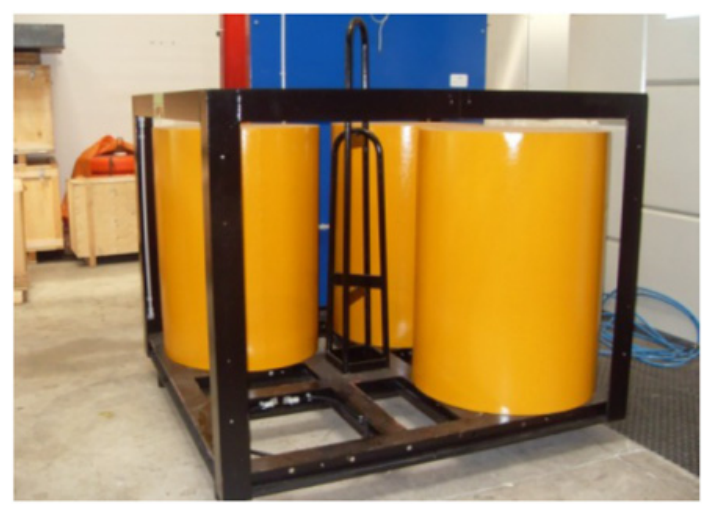

Figure 1. Pallet of (mock-up) drums, constructed at INFN-LNS for test purposes.

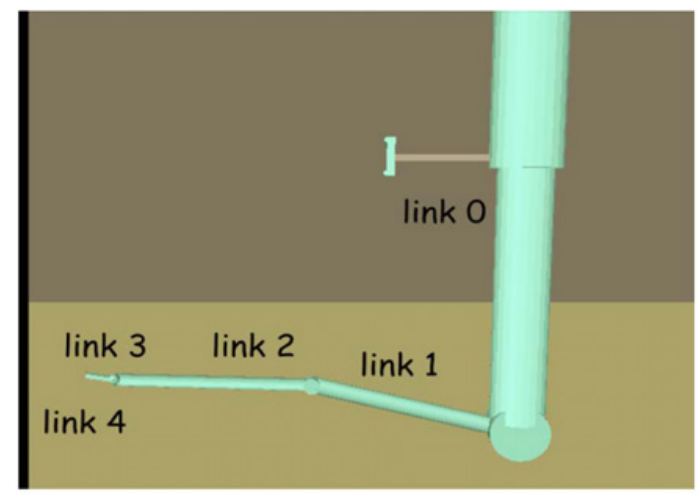

Figure 2. 3D sketch of the arm configuration.

The first part of the work is aimed at the manipulator design, which depends on the foreseen geometrical structure the arm will be operating in. In our case the "pallet" of drums is composed of an metal structure similar to the one shown in Fig. 1.

In this context a manipulator is presented with particular features (Fig. 2) suitable to skip obstacles and to explore all parts of the drums. It consists of five links: a fixed link and four revolute joints (two for the end-effector) [5].

A fixed frame in the upper part of the manipulator is to be connected to a crane allowing for the vertical motion.

\section{Simulation of the arm}

The determination of the frame of reference of a joint, according to the Denavit-Hartemberg convention [4], gives us the possibility to find the transformation matrix, which represents the transition from the frame of reference to the next one. So it is possible to determine the position of the endeffector simply considering a rotation matrix, which is expressed as a succession of partial rotations. Each rotation is defined with respect to the previous one (rotation in current reference frame). The composition of successive rotations with respect to the current frame is obtained by multiplying from left to right the individual rotation matrices, in the order of rotation. This is called direct kinematics. 


\section{$3^{\text {rd }}$ European Energy Conference}

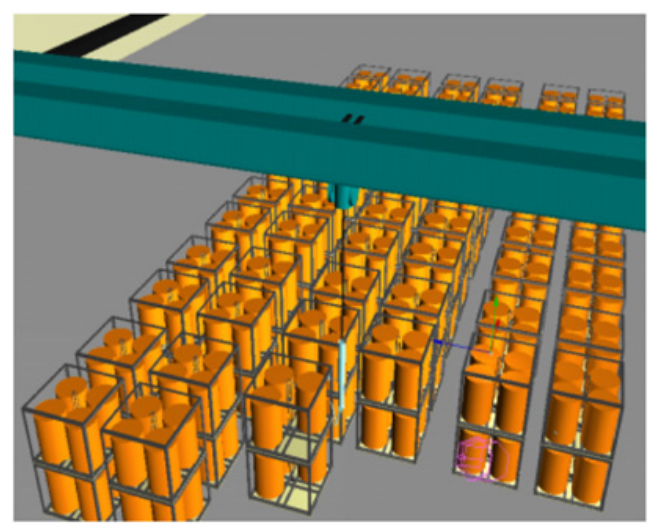

Figure 3. Sketch of the arm being moved around a temporary storage site by the crane.

The inverse kinematics problem allows to determine the value of the joint variables from the position and orientation of the end-effector.

The direct kinematics admits always a solution, the inverse kinematic problem could have multiple solutions, i.e. multiple ways to position each link. It may happen to have infinite solutions to the inverse kinematic problem and in such a case one speaks about "redundant manipulator" (number of links larger than the degrees of freedom - DOF).

In order to avoid this problem a new kinematic function was defined, following geometric considerations. This particular case is called two-link planar manipulator.

The equation implemented in MatLab [10] to find the angle for the link2 is:

$$
\theta_{2}= \pm 2 \tan ^{-1} \sqrt{\frac{\left(a_{1}+a_{2}\right)^{2}-\left(x^{2}+y^{2}\right)}{\left(x^{2}+y^{2}\right)-\left(a_{1}-a_{2}\right)^{2}}}
$$

while for the first link the following equations have been used:

$$
\begin{gathered}
\Phi=a \tan ^{2}(y-x) \\
\varphi=a \tan ^{2}\left(a_{2} \sin \theta_{2}, a_{1}+a_{2} \cos \theta_{2}\right) \\
\theta_{1}=\Phi-\varphi .
\end{gathered}
$$

The approximation of considering the problem as planar is acceptable, because in our case the manipulator moves along the $\mathrm{Z}$ and $\mathrm{X}$ axes, maintaining $\mathrm{Y}$ axis equal to 0 in every situation.

The model for the simulation was done in Simulink [11] (Fig. 5). A Manual Mode and an Automatic Mode were implemented following the previous mathematical considerations.

\section{RadIBot prototype}

After implementing the simulation, the real manipulator was built. The first idea of was focused on a robot with belts, that was not the best solution due to possible materials wear problems. Therefore a solution was devised that was more robust and versatile, but especially that better suited the characteristics of weight and size needed for the monitoring task at hand.

RadIBot, a robotic arm with five degrees of freedom, was then built with capabilities to take motion and still pictures of drums and to investigate possible leaks by means of a radiation sensor. 


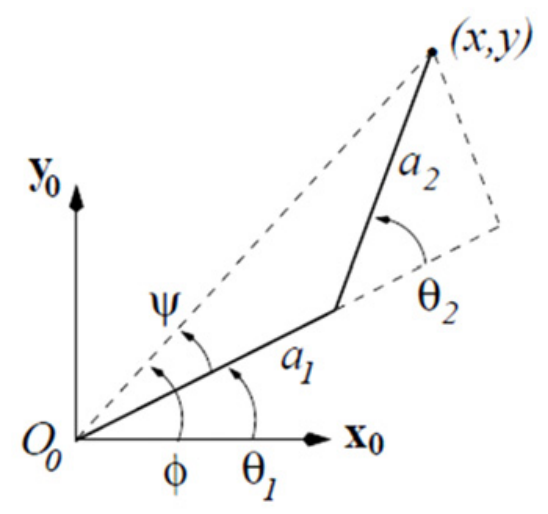

Figure 4. Inverse kinematic problem for a two link planar manipulator.

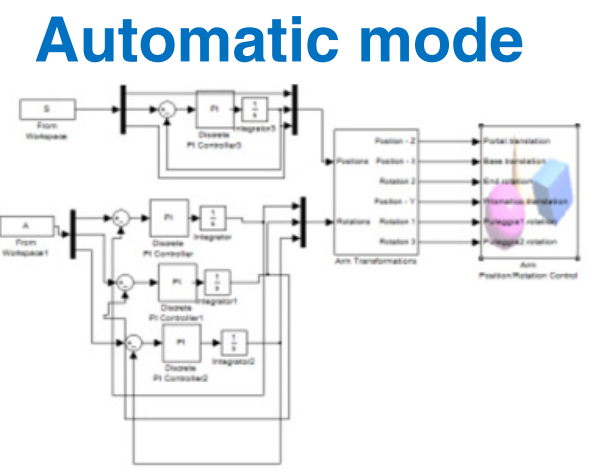

\section{Manual Mode}

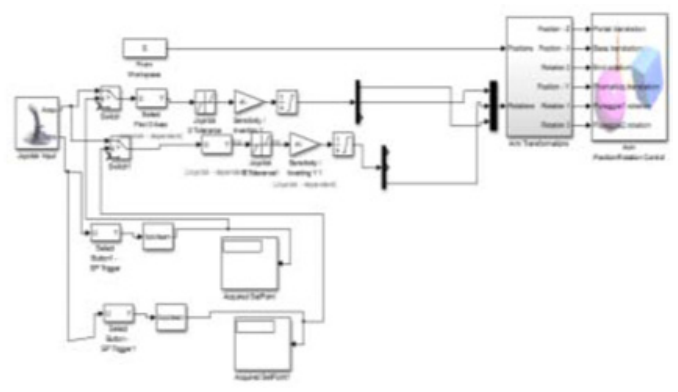

Figure 5. Simulink model of automatic and manual modes.

The overall structure is made from aluminum, thin and light, that hosts the engines. The robot has 3 links and one end-effector. The first link was initially designed as a prismatic joint, but the possibility to use a crane for the vertical motion suggested to implement it as a static link. The second and the third links were made in order to obtain two revolute joints. They must mechanically support the whole structure, each one being $25 \mathrm{~cm}$ long, $6 \mathrm{~cm}$ wide and weighing of $80 \mathrm{~g}$.

The link cross section is rectangular, and it is void to save weight. Figure 6 shows a drawing and the corresponding picture of the second and third link.

The choice of engines has been difficult because it had to solve the problem of the required high torque, in relation to the arm length $(65 \mathrm{~cm}$, without the static first link, and $1 \mathrm{~kg}$ of payload on the end effector). Two Dynamixel engines were chosen: MX106R and RX64 [6, 7]. These engines have been chosen for their possibility to use two of them in parallel to double the torque, through the dual mode joints.

As the manipulator might interact with an environment potentially harmful for the electronics, we decided to use engines with limited electronics. Indeed it is possible to connect Dynamixel engines in master-slave configuration (Fig. 7) with high level interfaces like LabView or MatLab, without additional electronics.

The computer can be considered the master that controls the motors, linked together, through 4-wire cables (slaves). This approach is perfect for a machine-operator system. Through the master the operator 

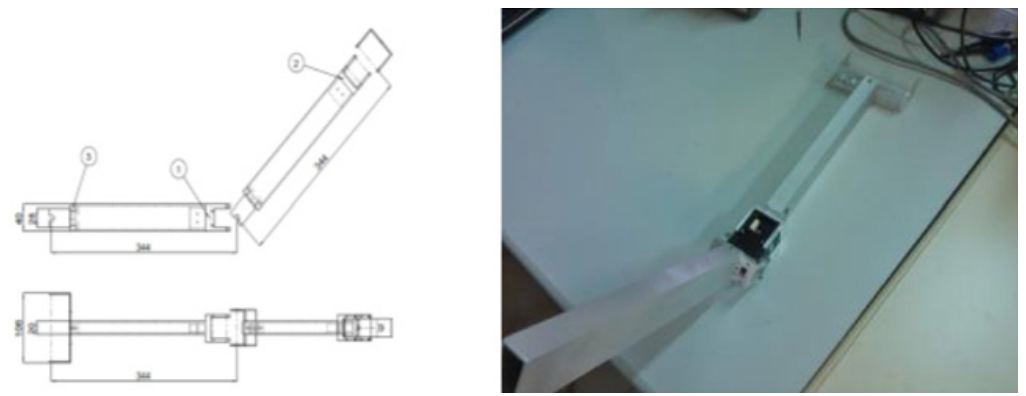

Figure 6. CAD sketch of the third and fourth links (lefthand side) and the corresponding picture (righthand side).

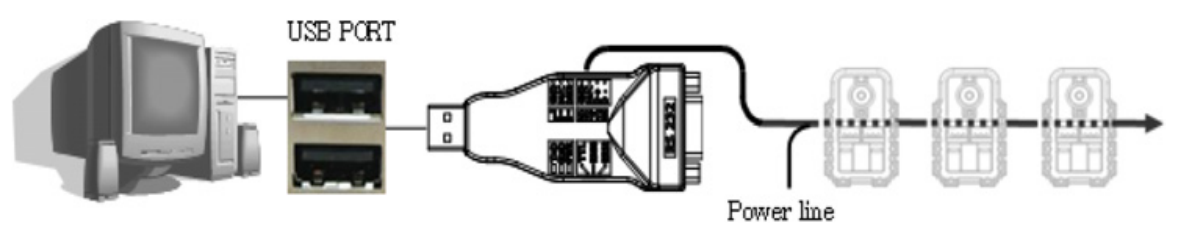

Figure 7. Dinamixel control using a PC.

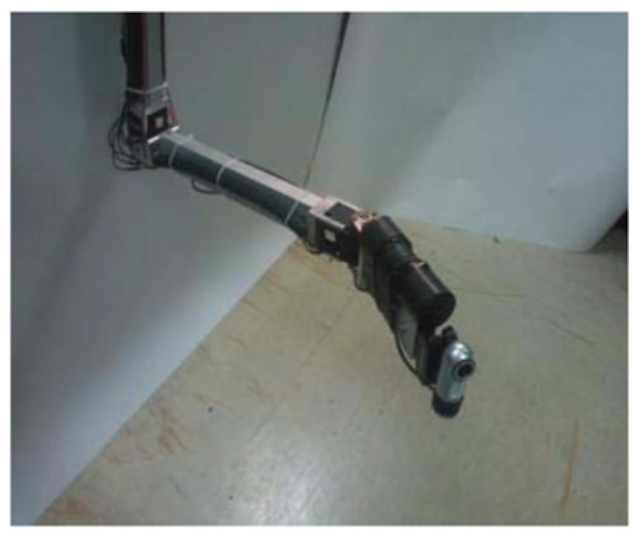

Figure 8. RadIBot detail of the last link and end-effector.

can collect relevant information or directly move/stop the manipulator. The communication between the engines and the PC has been realized by means of a serial communication protocol $[8,9]$.

The final prototype is shown in Fig. 8. On the end-effector we placed a compact videocamera meant to inspect the radwaste drums, plus a $\mathrm{CsI}(\mathrm{Tl})$ scintillator, coupled to a photomultiplier, acting as gammaray detector to follow up with finer measurements on the indications coming from the DMNR system.

\section{HMI interaction}

An operator interface was designed in MatLab to allow an easy interaction with the robot (). A friendly high-level interface is mandatory, in order to manage a tool like a robotic arm. Even without a particular radiation protection training, an operator can use the interface to move the robot around and and perform inspection activities. 


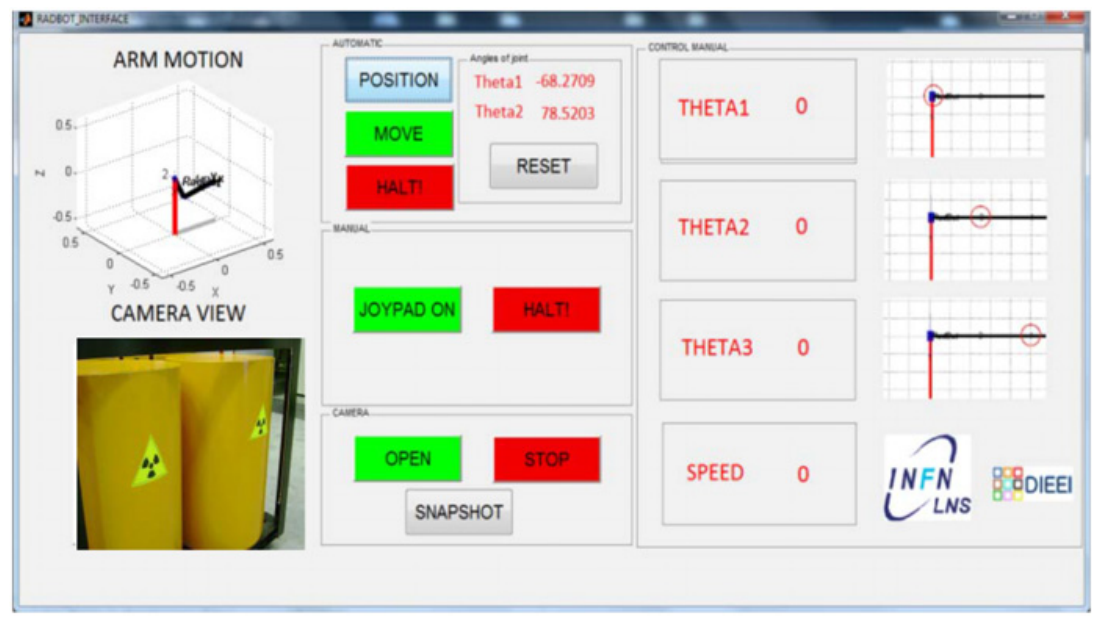

Figure 9. RadIBot operator interface.

The interface is divided into four panels:

- AUTOMATIC

- MANUAL

- CAMERA

- CONTROL MANUAL.

The AUTOMATIC section allows, according to the choice of a point in 2D space, the calculation of the optimal configuration and of the joint angles for inverse kinematics of a planar two-link manipulator.

The POSITION button opens a window that allows to choose the point to be investigated in the space. After the calculation of the joint angles, the angle values are plotted on the interface window in order to check the correctness of the kinematics and also the trajectory of the manipulator in the ARM MOTION output panel. The engines can be actuated through the MOVE button. The RESET button allows the manipulator to reach a reference starting position, that can be modified by the programmer, according to the foreseen workspace of the manipulator. The HALT! button is used for safety and immediately stops the manipulator.

The section MANUAL allows the operator a manual inspection. It is possible to choose the joint to be moved by means of a joypad (for our tests we used a cheap joypad with 4 axes and 10 buttons). Extreme care is required in this case, because the user manually decides the trajectories of the arm, that may invade the workspace and come in contact with obstacles. Even in this case the HALT! panic button can be used to stop the manipulator and prevent damage.

The section CAMERA has been designed to control the camera on the end effector of the robot. It is compatible with any USB camera on the market. By means of the three buttons one can start the camera, stop the camera and using the button SNAPSHOT it is possible to take a still picture of the current detail on the screen.

The section CONTROL MANUAL gives information about the status of the manipulator. Thetal, theta2, theta 3 and speed are the most important values to understand the status of each motor. It starts operating when the Joypad $\mathrm{ON}$ button is pressed, and the initial values of angles come from the Manual Mode. The three drawings of the manipulator on the rightmost part of the interface window help to understand the rotation of the link. 


\section{Conclusion}

This work has been addressed to the study and the realization of a robotic arm for radwaste inspection.

The problem of waste disposal is quite important at national and international level. Several associations finance projects in order to sensitize the younger generations, to understand how to solve the problems related to radwaste and how to find viable solutions.

Disposal sites are currently the only viable approach and it is implemented in several countries, even though the problem of reducing the risk for the radwaste operators and the surrounding population is still present.

A strategy of continuous monitoring is therefore recommended for an early detection of possible leaks and to prevent more severe accidents. In this framework a policy of periodic inspections without the physical presence of operators, along with on-request remote inspections in case of anomalous behavior signaled by the monitoring system, would be very welcome. The robotic arm is a tool that comes close to a human arm, most widely used for the remote manipulation of objects and to establish a direct contact between man and environment. As a safe replacement of a human arm, a robotic arm can represent a quite useful complementary tool to a remote monitoring system, in order to explore an environment dangerous for people.

\section{References}

[1] Luigi Cosentino, Claudio Calì, Giovanni De Luca, Giovanni Guardo, Pietro Litrico, Alfio Pappalardo, Massimo Piscopo, Carlotta Scirè, Sergio Scirè, Enrico Botta, and Paolo Finocchiaro, "Real-time online monitoring of radwaste storage: a proof-of-principle test prototype”, IEEE Transactions on Nuclear science, VOL. 59, NO. 4, AUGUST 2012

[2] Paolo Finocchiaro, "DMNR: A new concept for REAL-TIME online monitoring for short and medium term radioactive waste", Nova Science Publishers, Inc. 2011

[3] P. Finocchiaro , M. Barbagallo, L. Cosentino, G. Greco, G. Guardo, A. Pappalardo, C. Scirè, S. Scirè, "Silicon photomultipliers for radioactive waste online monitoring", Elsevier

[4] Mahesh D. Pandey, Xufang Zhang, "System reliability analysis of the robotic manipulator with random joint clearances", Mechanism and Machine Theory Volume 58, December 2012

[5] Carlotta Scirè Scappuzzo, "Studio di un sistema robotizzato per il monitoraggio di depositi di scorie radioattive", Master Thesis 2010/2011

[6] http://support.robotis.com/en/product/dynamixel/mx_series/mx-106.html

[7] http://support.robotis.com/en/product/dynamixel/rx_series/rx-64.html

[8] http://support.robotis.com/en/product/auxdevice/interface/usb2dxl_manual. html

[9] http://support.robotis.com/en/software/dynamixel_sdk/api_reference.html

[10] The MathWorks, Inc. MATLAB - The Language of Technical Computing, Version 2013a, The MathWorks, Inc., Natick, Massachusetts. URL http://www.mathworks. com/products/matlab/

[11] The MathWorks, Inc. SIMULINK - The MathWorks, Inc., Natick, Massachusetts. URL http://www . mathworks. com/products/simulink/ 